\title{
Medical hypothesis: speculating on the pathogenesis of acute diverticulitis
}

\author{
Angelo Zullo \\ Nuovo Regina Margherita Hospital, Rome, Italy
}

\begin{abstract}
The pathogenetic process of acute diverticulitis remains speculative. According to the most widely accepted theory, the mechanism involved is "traumatic" damage to the mucosa due to fecolith impaction, as occurs in large diverticula. However, not uncommonly, diverticulitis develops in young patients with only few and small diverticula, where fecolith trapping is very unlikely. Therefore, another theory is necessary to clarify this process. A possible explanation could be "ischemic" damage. According to this theory, an ischemic lesion is caused by the compression of vascular structures in the neck of the diverticular task, as a result of prolonged and/or recurrent contractile spikes related to neuromuscular alterations in the diverticular tract. Clearly, the "traumatic" and "ischemic" mechanisms of acute diverticulitis are not mutually exclusive, and may act in different patients. The existing data corroborating these theories are presented and different potential therapeutic approaches are briefly discussed.
\end{abstract}

Keywords Diverticulitis, pathogenesis, therapy

Ann Gastroenterol 2018; 31(6): 1-3

Acute diverticulitis is a complication that develops in nearly $4 \%$ of patients with diverticular disease (DD), a widespread condition in Western countries [1]. Diverticulitis consists in the inflammation of a diverticulum, which causes a combination of symptoms, including acute abdominal pain (typically in the lower right quadrant in Caucasian patients), fever and bowel movement alterations, whilst stool blood is generally absent or modest [2,3].

Although our knowledge of the natural history and management of diverticulitis has greatly increased, the real cause of diverticulitis onset still remains speculative. In the past, the inflammation was attributed to a primary infection of a diverticular task, potentially leading to a perforation, although no specific pathogens have been demonstrated to cause diverticulitis. Consequently, all patients with acute, uncomplicated diverticulitis were treated with a combination of broad-spectrum antibiotics (generally third-generation cephalosporin or quinolones), together with metronidazole to overcome anaerobic bacteria [4].

Gastroenterology and Digestive Endoscopy, Nuovo Regina Margherita Hospital, Rome, Italy

\section{Conflict of Interest: None}

Correspondence to: Dr. Angelo Zullo, Gastroenterologia ed Endoscopia Digestiva, Ospedale Nuovo Regina Margherita, Via Emilio Morosini 30, 00153 Rome, Italy, e-mail: angelozullo66@yahoo.it

Received 4 September 2018; accepted 9 September 2018; published online 26 September 2018

DOI: https://doi.org/10.20524/aog.2018.0315
Currently, diverticulitis is considered to be an acute inflammation caused by a "traumatic" damage of a diverticulum by stool impaction, followed by bacterial proliferation, similarly to that of appendicitis [2-8]. It has been suggested that the increased colonic pressure in DD is responsible for pushing fecoliths into the diverticular task, particularly in large diverticula. Thus, the trapped fecolith abrades the mucosa of the diverticular sac, causing inflammation and expansion of usual bacterial flora. Subsequently, bacteria may breach the mucosa and extend the process through the full wall thickness, where toxin production, gas production and the mucosal injury ultimately lead to perforation [5]. Alternatively, the traumatic damage from a fecolith may cause irritation of the mucosa with low-grade inflammation, then vascular congestion and further obstruction of the diverticular tract leading to stool trapping and bacterial overgrowth, with purulent material forming inside the diverticular sac [8]. Such a hypothesis is, at least in part, corroborated by the sequence observed on ultrasonography of fecolith obstruction, pus formation, spontaneous fecolith expulsion, and resolution in selected cases [9]. According to this theory, the role of bacterial overgrowth appears to be prominent, so that antibiotic therapy is actually considered the cornerstone therapy for acute diverticulitis. However, in the last decade, various studies have shown that acute uncomplicated diverticulitis may be safely managed without standard antibiotic therapy, at least in selected patients [10]. However, further long-term, randomized clinical trials will be required before we change our clinical practice.

In the meantime, another theory of acute diverticulitis pathogenesis may be put forward: the so-called "ischemic" 
damage, in which fecoliths play no role. In reality, this theory fits particularly well with diverticulitis onset in diminutive diverticula, where the trapping of fecoliths and/or other material, such as alimentary seeds, is unlikely. Indeed, the role of seeds in diverticulitis-first theorized by Horner in 1958 [11]-has never been confirmed in clinical practice. On the contrary, data from a large cohort study indicated an inverse correlation between the consumption of seeds and nuts and diverticulitis onset [12]. The trigger of the ischemic process could be a long-lasting (or recurrent) compression of vasa recta in the "neck" of the diverticulum due to a prolonged and/or marked contractile spike of the colon. Indeed, the tiny "neck" of the diverticulum passes through the circular muscle of the bowel wall and may be abnormally compressed, leading to mucosal ischemia at the apex of the sac or even microperforation. Some experimental and clinical observations may support this hypothesis. In one study, DD patients showed a significantly greater increase of forceful propulsive activity in affected segments compared with controls, with abnormal retrograde propagated activity in $20 \%$ of cases [13]. Likewise, these findings depend on well-documented neuromuscular alterations in the diverticular tract. creating hypersensitivity due to a cholinergic denervation. Indeed, lower choline acetyltransferase activity, M3 receptor upregulation and increased sensitivity to exogenous acetylcholine have been demonstrated in DD patients compared with controls [14]. Therefore, the sigmoid wall with diverticula responds with exaggerated and prolonged contractile spikes to normal stimuli, so that abnormal compression of the small vasculature penetrating into the diverticulum is not unrealistic. Regarding clinical support, diverticulitis mainly develops in patients with symptomatic uncomplicated DD [2,3]. It has been found that $22 \%$ of these patients suffer from episodes of lower left quadrant abdominal pain lasting $>24 \mathrm{~h}$ [15]. Independently of the cause, such a condition is sustained, at least in part, by a a persistent spastic state in the diverticular tract [6], potentially predisposing to vascular compression and mucosal ischemia in the diverticulum. On the other hand, it is a common experience to manage acute diverticulitis in young patients with only few (seldom just one) and small diverticula. In these cases, fecal impaction may be reasonably excluded and an ischemic process occurring in the small diverticula orifice due to a prolonged spasm of bowel wall involving the diverticular neck could be advocated, similar to a strangulated inguinal hernia. Therefore, this process may develop with or without secondary infection from fecal bacteria.

Clearly, the "traumatic" and "ischemic" mechanisms of acute diverticulitis are not mutually exclusive, and they may act in different patients or in different diverticula. The former process is the most frequent pathogenesis in elderly patients with several large diverticula configuring a tortuous, thickened, and narrowed sigmoid tract. The latter may be mainly involved in diverticulitis of young patients with sparse and diminutive diverticula. The function of fecal bacteria may also be different. It is a constant phase according to the "traumatic" theory, whilst it is secondary (or absent) in the ischemic process (Fig. 1). Therefore, the role of bacterial infection in the pathogenesis of acute diverticulitis deserves a reappraisal. Most probably, we are administering systemic antibiotics in patients with acute diverticulitis as prevention rather than curing an established infection. Such a cautious approach is justified by the presence of billions of (potentially aggressive) bacteria in the colon, so that antibiotic therapy is mainly intended to prevent an opportunistic infection on a damaged mucosa, rather than
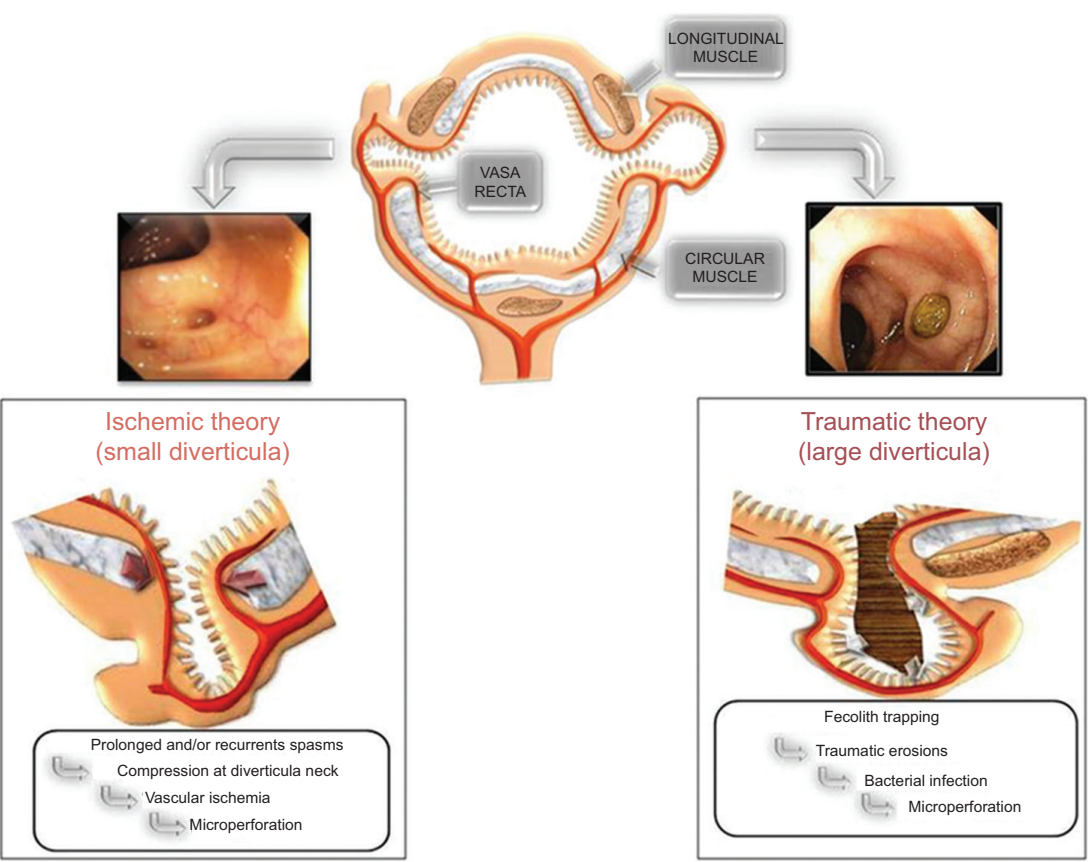

Figure 1 The flow-chart describing "traumatic" and "ischemic" theories for diverticulitis 
curing an ongoing infection. On the other hand, we adopt the same approach to prevent an infection following a surgical intervention, as well as other medical maneuvers with potential infective risk.

At least in theory, the two different processes of diverticulitis may benefit from diverse therapeutic approaches. The use of fibers in DD subjects, as well as other agents preventing constipation, could be useful for reducing the risk of fecolith formation. On the other hand, early administration of spasmolytic agents is expected to be useful in patients with pain lasting $>24 \mathrm{~h}$ in order to promptly interrupt the cascade leading to ischemic damage. Experimental data have clearly demonstrated that hyper-contractions of circular muscle in the sigmoid tract with DD are reduced by anticholinergic drugs [16], altough higher dose may by necessary [17].

In conclusion, there are two theories to explain acute diverticulitis onset: the "traumatic" and the "ischemic" mechanism. These processes are not mutually exclusive, and may act in different patients.

\section{References}

1. Stollman N, Smalley W, Hirano I; AGA Institute Clinical Guidelines Committee. American Gastroenterological Association Institute Guideline on the Management of Acute Diverticulitis. Gastroenterology 2015;149:1944-1949.

2. Stollman N, Raskin JB. Diverticular disease of the colon. Lancet 2004;363:631-639.

3. Jacobs DO. Clinical practice: Diverticulitis. $N$ Engl $J$ Med 2007;357:2057-2066.

4. Tursi A. Advances in the management of colonic diverticulitis. CMAJ 2012;184:1470-1476.
5. West AB; NDSG. The pathology of diverticulitis. JClin Gastroenterol 2008;42:1137-1138.

6. Humes DJ, Spiller RC. Review article: the pathogenesis and management of acute colonic diverticulitis. Aliment Pharmacol Ther 2014;39:359-370.

7. Vermeulen J, van der Harst E, Lange JF. Pathophysiology and prevention of diverticulitis and perforation. Neth $J$ Med 2010;68:303-309.

8. Rezapour M, Ali S, Stollman N. Diverticular disease: an update on pathogenesis and management. Gut Liver 2018;12:125-132.

9. Puylaert JB. Ultrasound of colon diverticulitis. Dig Dis 2012;30:5659.

10. van Dijk ST, Bos K, de Boer MGJ, et al. A systematic review and meta-analysis of outpatient treatment for acute diverticulitis. Int $J$ Colorectal Dis 2018;33:505-512.

11. Horner JL. Natural history of diverticulosis of the colon. Am J Dig Dis 1958;3:343-350.

12. Strate LL, Liu YL, Syngal S, Aldoori WH, Giovannucci EL. Nut, corn, and popcorn consumption and the incidence of diverticular disease. JAMA 2008;300:907-914.

13. Bassotti G, Battaglia E, Spinozzi F, Pelli MA, Tonini M. Twenty-four hour recordings of colonic motility in patients with diverticular disease: evidence for abnormal motility and propulsive activity. Dis Colon Rectum 2001;44:1814-1820.

14. Golder M, Burleigh DE, Belai A, et al. Smooth muscle cholinergic denervation hypersensitivity in diverticular disease. Lancet 2003;361:1945-1951.

15. Cuomo R, Barbara G, Andreozzi P, et al. Symptom patterns can distinguish diverticular disease from irritable bowel syndrome. Eur J Clin Invest 2013;43:1147-1155.

16. Maselli MA, Piepoli AL, Guerra V, et al. Colonic smooth muscle responses in patients with diverticular disease of the colon: effect of the NK2 receptor antagonist SR48968. Dig Liver Dis 2004;36:348354.

17. Alvarez-Berdugo D, EspIn F, Arenas C, et al. Changes in the response to excitatory antagonists, agonists, and spasmolytic agents in circular colonic smooth muscle strips from patients with diverticulosis. Neurogastroenterol Motil 2015;27:1600-1612. 